\title{
Pleural Fluid with Hooklets in a 7-year-old Boy with Severe Multisystem Cystic Echinococcosis
}

Patrick M. Meyer Sauteur, MD PhD ${ }^{1,}{ }^{\star}$, Sasha J. Tharakan, $\mathrm{MD}^{2}$, Christa Relly, MD${ }^{1}$, Felicia Drack, $\mathrm{MD}^{3}$, Ralph A. Schmid, MD ${ }^{4}$, Kevin Schmid, MD ${ }^{5}$, Alexander Moeller, MD ${ }^{6}$, Ralph Gnannt, MD , Christoph Berger, MD¹, Ueli Moehrlen, MD²

1 Division of Infectious Diseases and Hospital Epidemiology, University Children's Hospital Zurich, Zurich, Switzerland

2 Department of Pediatric Surgery, University Children's Hospital Zurich, Zurich, Switzerland

3 Emergency Department, University Children's Hospital Zurich, Zurich, Switzerland

4 Department of General Thoracic Surgery, Bern University Hospital, University of Bern, Switzerland

5 Intensive Care Unit, University Children's Hospital Zurich, Zurich, Switzerland

6 Division of Respiratory Medicine, University Children's Hospital of Zurich, Switzerland

7 Division of Diagnostic Imaging, University Children's Hospital Zurich, Zurich, Switzerland

* Corresponding author:

Patrick M. Meyer Sauteur, MD PhD 
Division of Infectious Diseases and Hospital Epidemiology, University Children's Hospital Zurich, Steinwiesstrasse 75, CH-8032 Zurich, Switzerland

Phone: +41 4426678 96; E-mail: patrick.meyer@kispi.uzh.ch

Short running head: Severe childhood multisystem cystic echinococcosis

Word count: 388

\section{ORCID IDs}

Patrick M. Meyer Sauteur, MD PhD 0000-0002-4312-9803

Sasha J. Tharakan, MD 0000-0001-6961-1174

Christa Relly, MD NA

Felicia Drack, MD 0000-0002-4580-6206

Ralph A. Schmid, MD 0000-0003-0699-079X

Kevin Schmid, MD NA

Alexander Moeller, MD 0000-0001-7284-4251

Ralph Gnannt, MD $\quad$ 0000-0001-8120-6200

Christoph Berger, MD 0000-0002-2373-8804

Ueli Moehrlen, MD 0000-0001-6418-1136

\section{Author's contributions}

We were all involved in the patient's care and diagnosis. P.M.M.S. wrote the first draft of the manuscript. We all edited the manuscript and approved the final version. Written consent for publication was obtained from the patient's father.

\section{Funding}

None. 


\section{Conflict of interest}

None.

\section{Subject Category List}

10.11 Pediatrics: Respiratory Infections

\section{Online Data Supplement}

This article has an online data supplement, which is accessible from this issue's table of content online at www.atsjournals.org.

\section{Acknowledgments}

We would like to thank Claudia Steiger (Hematology Laboratory, University Children's Hospital Zurich, Switzerland) for the microscopy pictures, and the Institute of Parasitology (Vetsuisse and Medical Faculty, University of Zurich, Switzerland) for performing the specific PCR assay and serology. 
A 7-year-old boy from Macedonia who immigrated to Switzerland two years earlier presented to our clinic with a cough since a week and loss of appetite for months. His medical history was unremarkable and he was not exposed to animals. On physical examination, he was tachypneic and had absent breath sounds on the right hemithorax. Laboratory investigations revealed a total leukocyte count of $11,610 / \mu l$ without eosinophilia and a C-reactive protein level of $103 \mathrm{mg} / \mathrm{L}$. Chest $\mathrm{x}$-ray showed opacification of the right hemithorax (Figure $1 \mathrm{~A}$ ) with $>10 \mathrm{~mm}$ rim of fluid on sonography pointing at a large pleural effusion (1). A diagnostic pleural puncture for suspected parapneumonic effusion drained $40 \mathrm{~mL}$ of clear fluid. Gram stain and antigen testing for Streptococcus pneumoniae on pleural fluid specimens were negative. Subsequently, the patient developed a pneumothorax (Figure 1B), requiring tracheal intubation and pleural needle decompression. Computed tomography revealed cystic masses in the right hemithorax (Figure $1 \mathrm{C}$ ) and the liver (Figure 1D).

Pleural fluid microscopy showed protoscoleces from Echinococcus granulosus (Figure 1E) with shiny colorful hooklets (Figure 1F and Video 1). Cystic echinococcosis was confirmed by polymerase chain reaction and serology (enzymelinked immunosorbent assay and immunoelectrotransfer blot) (2). Pleural fluid and blood cultures and a tuberculin skin test were negative. The accidental cyst puncture with spillage of protoscoleces led to anaphylaxis and acute respiratory distress syndrome requiring extracorporeal membrane oxygenation for 4 days. He was started on albendazole, followed by urgent lobectomy of the right upper lobe, total pericystectomy of the right lower lobe at 3 weeks (Figure E1), and puncture, aspiration, injection, re-aspiration (PAIR) technique of the liver cyst at 3 months. Albendazole was administered for total 5 months. A 6-month follow-up revealed 
normal level of activity but exertional dyspnea, re-expansion of the right lower lobe, and reduction in the size of the liver cyst.

Cystic echinococcosis is a very rare disease in children but may be considered as differential diagnosis of pneumonia with pleural effusion in migrant children from endemic areas (3-5). Even though the incubation period lasts for years also young children can be affected (3). Our case further highlights that symptoms in children may be sparse or lacking despite enormous mass effect of cysts (3). Hooklets on microscopy can be clues to diagnosis $(6,7)$. Importantly, spillage of protoscoleces should be avoided to prevent anaphylaxis and severe immunological reactions (8). 


\section{References}

1. Bradley JS, Byington CL, Shah SS, Alverson B, Carter ER, Harrison C, Kaplan SL, Mace SE, McCracken GH, Jr., Moore MR, St Peter SD, Stockwell JA, Swanson JT, Pediatric Infectious Diseases S, the Infectious Diseases Society of A. The management of community-acquired pneumonia in infants and children older than 3 months of age: clinical practice guidelines by the Pediatric Infectious Diseases Society and the Infectious Diseases Society of America. Clin Infect Dis 2011;53:e25-76.

2. Schweiger A, Grimm F, Tanner I, Mullhaupt B, Bertogg K, Muller N, Deplazes P. Serological diagnosis of echinococcosis: the diagnostic potential of native antigens. Infection 2012;40:139-152.

3. Nourkami-Tutdibi N, Simon A, Fries P, Becker SL, Ecker P, Zemlin M, Meyer S. A 5-year-old boy with a chronic cough caused by Echinococcus granulosus. Lancet 2019;393:74.

4. McManus DP, Zhang W, Li J, Bartley PB. Echinococcosis. Lancet 2003;362:12951304.

5. Tersigni C, Venturini E, Montagnani C, Bianchi L, Chiappini E, de Martino M, Galli L. Should pediatricians be aware of cystic echinococcosis? A literature review. J Pediatr Gastroenterol Nutr 2019;68:161-168.

6. Brainard DM, Ryan ET. Thoracic echinococcosis. N Engl J Med 2003;348:528.

7. Sharma P, Kumar N, Jain A, Raghav N. Giant pulmonary hydatid cyst: an unusual presentation. Am J Respir Crit Care Med 2016;194:1026-1027. 
8. Brunetti E, Kern P, Vuitton DA, Writing Panel for the W-I. Expert consensus for the diagnosis and treatment of cystic and alveolar echinococcosis in humans. Acta Trop 2010;114:1-16. 
Figure 1. Severe childhood multisystem cystic echinococcosis. (A) Chest x-ray at presentation showing opacification of the right hemithorax, and $(B)$ after pleural puncture showing a thin-walled radiolucent lesion with curvilinear septa ("water lily sign", red arrow) in the right upper lobe, a radiopaque mass in the right lower lobe, and air trapping in the right pleural space with contralateral mediastinal displacement indicative of a tension pneumothorax. (C) Computed tomographic scan with contrast medium showing detached free-floating membranes within the low-density cystic lesion ("water lily sign", red arrow), a high-density cystic mass in the right lung (blue arrow), and (D) a high-density cystic mass in liver segment VIII (green arrow). (E) Light microscopy of pleural fluid showing everted protoscolex from Echinococcus granulosus (100x magnification, unstained wet preparation) with calcareous corpuscles (black arrow) and row of hooklets (insert shows corresponding area with magnification). (F) Phase-contrast microscopy with detailed view of hooklets (400× magnification, unstained wet preparation).

Video 1. Active movements of hooklets of Echinococcus granulosus in pleural fluid (400x magnification, unstained wet preparation). 

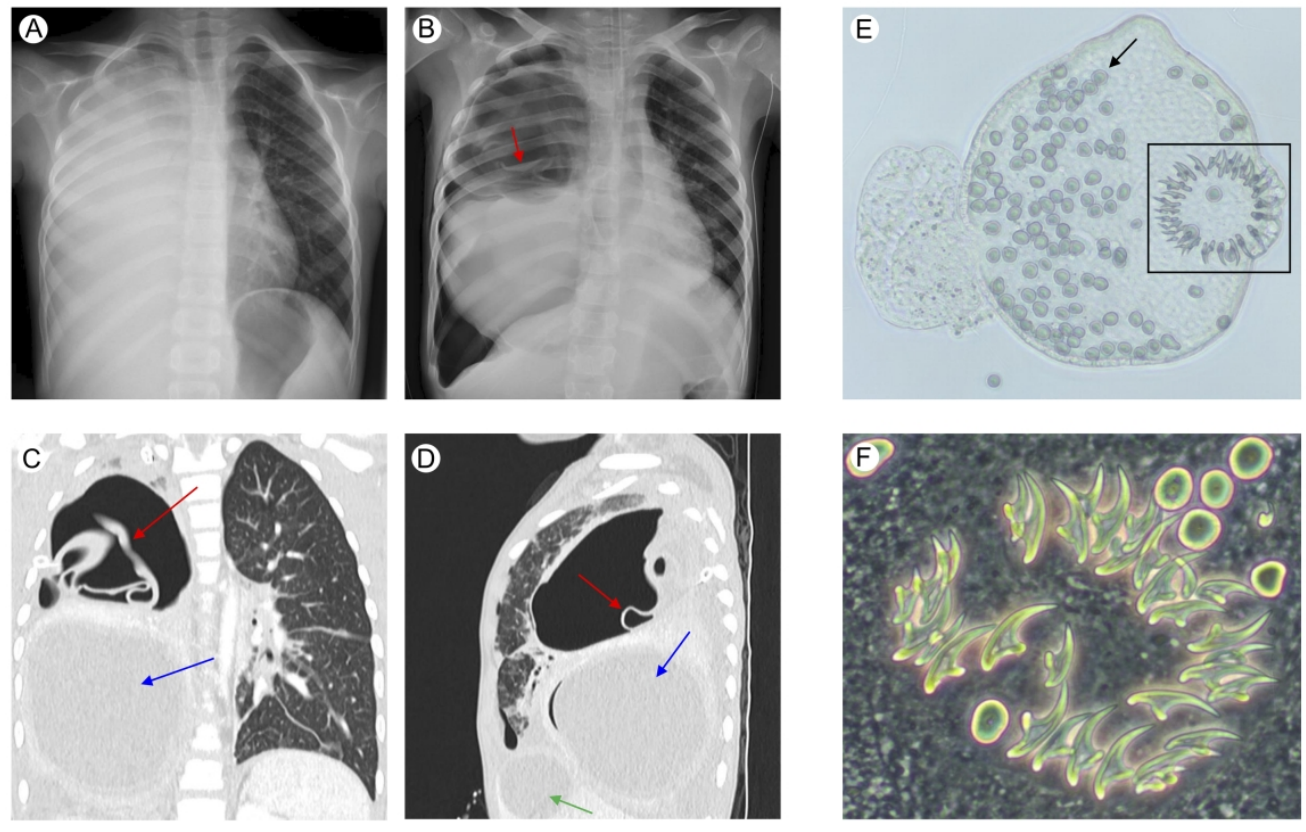

Figure 1.

$242 \times 153 \mathrm{~mm}(300 \times 300 \mathrm{DPI})$ 


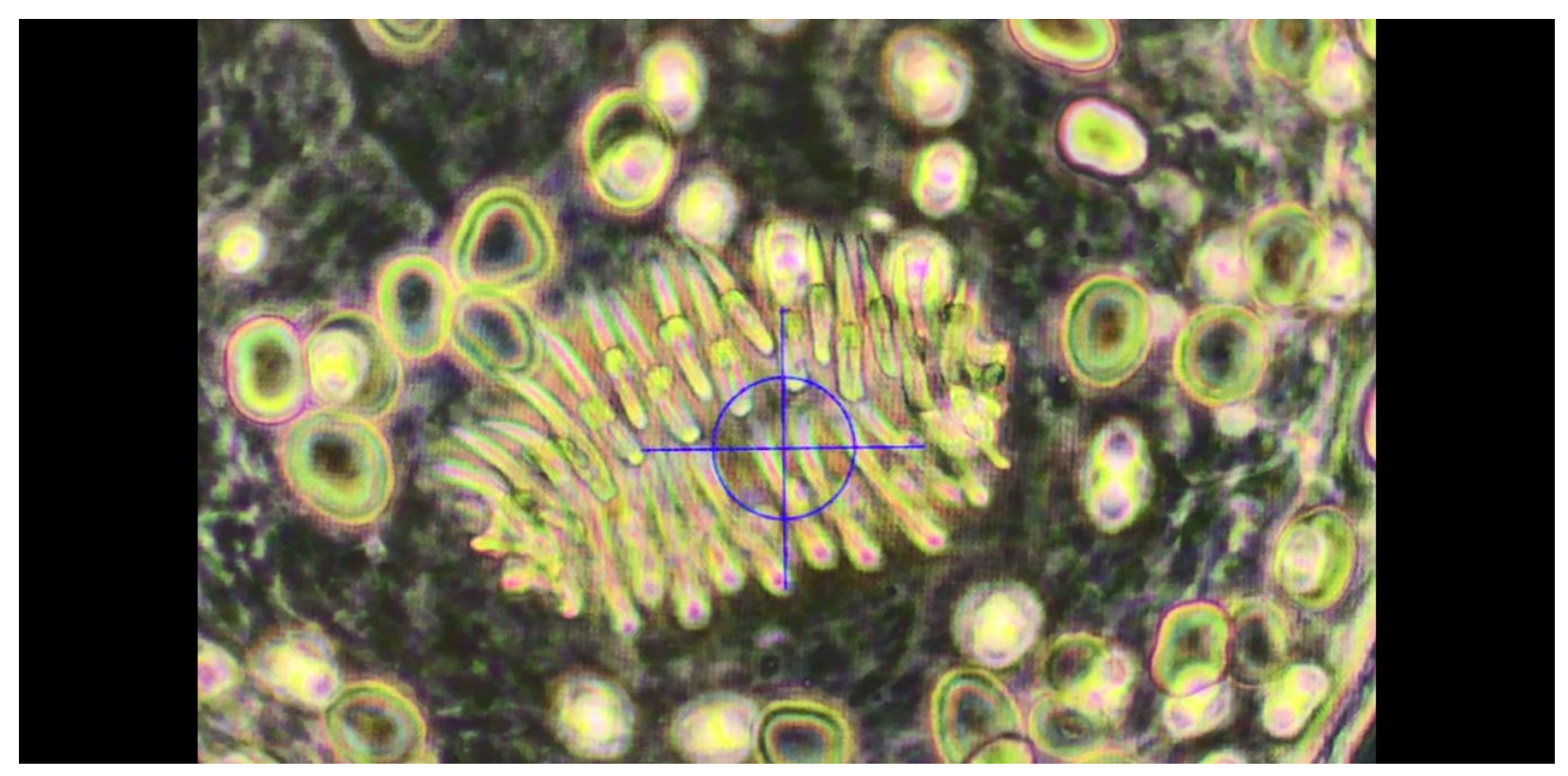

Video Still 


\section{Online Data Supplement}

Figure E1. Total pericystectomy of the right lower lobe. 


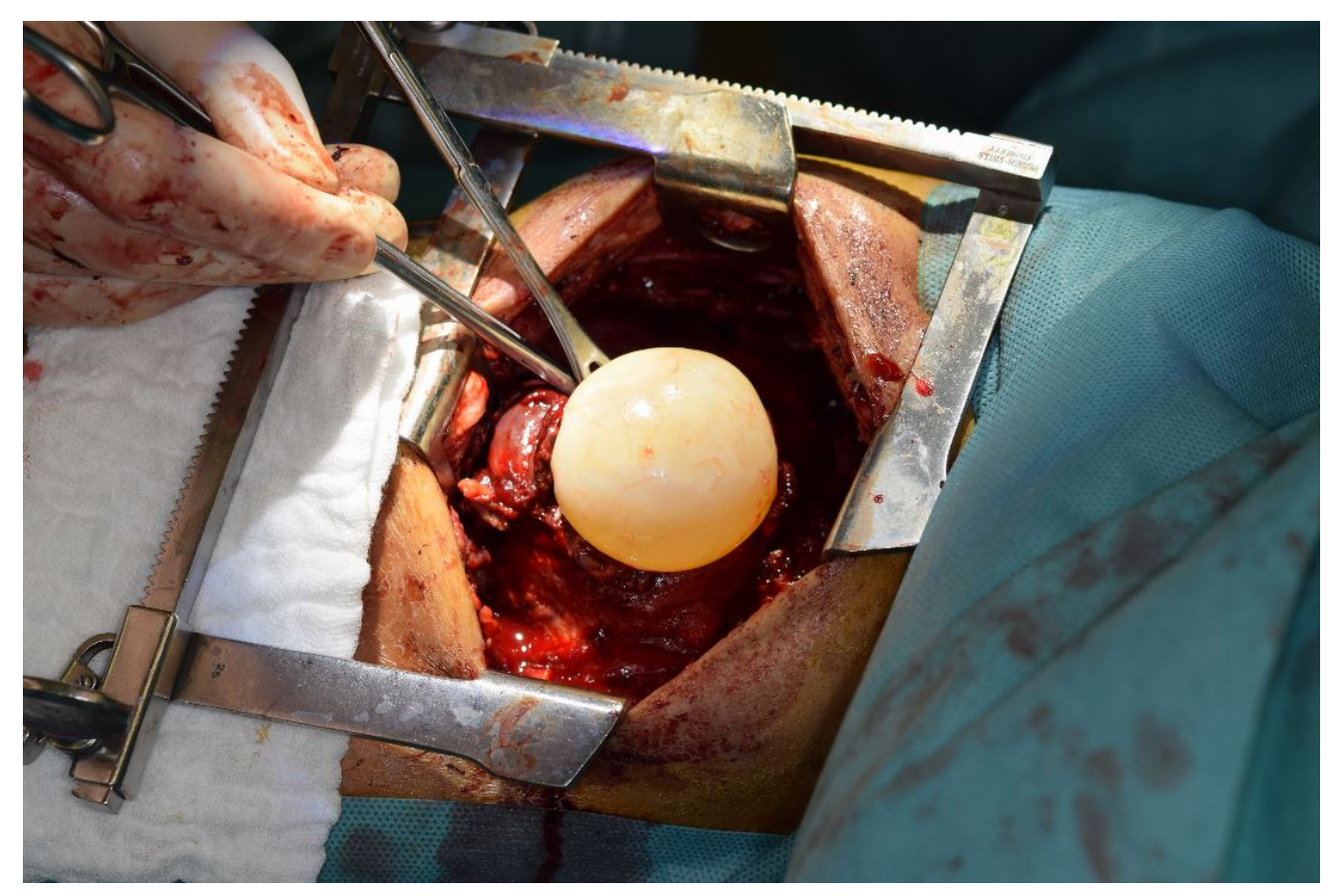

Figure E1. 